Kai Ambos

\title{
Artikel 7 EMRK, Common Law und die Mauerschützen
}

Die Europäisierung des (Straf-)Rechts wirft die bislang zu wenig diskutierte Frage nach den relevanten menschenrechtlichen Standards und damit zugleich Grenzen europarechtlicher Harmonisierung auf. Dem Gesetzlichkeitsprinzip (nullum crimen, nulla poena sine lege) kommt dabei eine herausragende Bedeutung zu, weil es - jedenfalls nach seiner theoretischen Konzeption - staats- und insbesondere strafrechtsbegrenzend wirken soll. Sein europarechtliches Verständnis in der Form von Art. 7 EMRK beruht jedoch auf dem common law (I., II.) und bleibt hinter Art. 103 II GG zurück. Die Bestätigung der deutschen Mauerschützenrechtsprechung durch den EGMR war damit zu erwarten, überzeugt aber in der Begründung nicht (III.).

\section{Common Law und »principle of legality»}

Common law ${ }^{1}$ ist richterrechtliches Gewohnheitsrecht, das sich ab der Mitte des 13. Jh. gegenüber dem lokalen county custom durchsetzte und damit das geltende Recht für alle Untertanen der englischen Krone - »common to the free people of England «wurde. ${ }^{2}$ Common Law ist also »judge made law « auf der Grundlage überlieferter Sitten und Gebräuche. Die Gerichte setzen oder artikulieren common law, wobei die Grenze zwischen konstitutiver Rechtssetzung und deklaratorischer Rechtsauslegung nur schwer zu ziehen ist. Klar ist, dass die ratio decidendi höchstrichterlicher Entscheidungen nach der Regel des precedent Bindungswirkung entfaltet, ${ }^{3}$ doch unklar ist häufig, was das geltende Recht ist.

In unserem Zusammenhang geht es - mit Blick auf Art. 7 EMRK - um das britische Recht, ${ }^{4}$ denn Großbritannien (England/Wales, Schottland und Nordirland) ist nicht nur die Wiege des common law, sondern auch Vertragspartei der EMRK; diese ist seit dem Human Rights Act 1998 auch Bestandteil des innerstaatlichen Rechts. Zudem

1 Vgl. Black's Law Dictionary, 7th edition, St. Pauls/ Minn. 1999, S. 270; Osborn's Concise Law Dictionary (Rutherford \& Bone eds.) 8. Aufl. 1993, S. 77; von Bernstorff, Einführung in das englische Recht, 1996, S. 2; auch Boot, Nullum crimen sine lege and the subject matter jurisdiction of the International Criminal Court, 2002, S. 112 Fn. 130.

$2 \mathrm{Zu}$ unterscheiden vom »gemeinen Recht« i.S.d. römischen (allgemeingültigen) Rechts, vgl. Fuller, Anatomy of the Law, 1968, S. 85.

3 Ebenso auf supranationaler Ebene im Verhältnis von Appeals Chamber zu den Trial Chambers der UN-Ad Hoc Tribunal, vgl. Prosecutor v. Aleksovski, Judgement 24 March 2000 (IT-95-14/1-A), para. 113. Schwächer hingegen Art. 21 Abs. 2 Rome Statute of the International Criminal Court (BGBl. 2000 II 1393), vgl. Ambos, Der Allgemeine Teil des Völkerstrafrechts, 2002, S. 49 m.w.N.

4 Dort ist common law eine der drei überlieferten Rechtsquellen, neben equity und Gesetzgebung (Black's, o. Fn. 1, S. 270; zur equity von Bernstorff, o. Fn. 1, S. 4 ff.). 
liegt in Großbritannien bis heute keine Gesamtkodifikation des Strafrechts vor, ${ }^{5}$ sondern das strafrechtliche statute law beschränkt sich auf Teilbereiche des Allgemeinen ${ }^{6}$ und vor allem des Besonderen Teils. ${ }^{7}$

Demgegenüber ist das Common Law in den ehemaligen Kolonien, vor allem den USA, zunehmend und praktisch vollkommen durch Statute Law verdrängt worden. ${ }^{8}$

Handelt es sich nun beim common law jedenfalls nicht um Recht in Form von Gesetzgebung (statute law), sondern allenfalls um die richterliche Festschreibung gewohnheitsrechtlich anerkannter Regeln, also um geschriebenes Richterrecht, ${ }^{9}$ scheint es $»$ necessarily retrospective in character ${ }^{10}{ }^{10}$ und gerät jedenfalls a limine mit der im kontinentalen Verständnis verbreiteten lex scripta Komponente des nullum crimen Grundsatzes in Konflikt. Freilich umfasst der Begriff »lex« auch nach völkerund europarechtlichem Verständnis ungeschriebenes Recht.

Insoweit sei hier nur, was das Völkerrecht angeht, auf die Arbeiten der UNVölkerrechtskommission zum Draft Code of Crimes against the Peace and Security of Mankind ${ }^{11}$ sowie den Bericht des UN-Generalsekretärs zur Errichtung des Jugoslawientribunals verwiesen, wonach (strafrechtliche) »rules«, die »beyond any doubt part of customary law $\ll$ sind, ${ }^{12}$ nicht den Nullum-crimen-Grundsatz verletzen. Was das europäische Recht angeht, so ist es st. Rspr. des EGMR, dass der Begriff Recht (»law «) in Art. 7 EMRK auch ungeschriebene Normen umfasse. ${ }^{13}$

Im common law wird der Nullum crimen Grundsatz in der Regel (nur) in einem dreifachen Sinne - als Rückwirkungsverbot, Bestimmtheitsgrundsatz und Analogieverbot (»non-retroactivity, maximum certainty and restrictive construction $\ll)^{14}-$ verstanden. Aus kontinentaleuropäischer Sicht handelt es sich damit um ein nullum

5 Vgl. aber den Vorschlag der Law-Commission Nr. 177, Criminal Law: Criminal Code for England and Wales (2 Bände) 1989; dazu auch Ashworth, Principles of Criminal Law, 3. Aufl. 1998, S. 7.

6 Etwa den Versuch (Criminal Attempts Act 1981) oder die Teilnahme (Accessories and Abettors Act 1861).

7 Nach Ashworth, Principles, o. Fn. 5, S. 6 fand die »major consolidation« 1861 statt; heute sei der Besondere Teil üw. kodifiziert (außer etwa manslaughter, murder, assault), doch insbesondere allgemeine Zurechnungsregeln, etwa intention, recklessness und defences (duress, intoxication, insanity), beruhten noch auf common law.

8 Für die USA vgl. La Fave/Scott, Criminal Law, 2. Aufl. 1986, S. 64 ff.

9 Will man nicht ohnehin das case law nur als Beleg für und Ausdruck von common law verstehen, vgl. zur Problematik La Fave/Scott, o. Fn. 8, S. 64 Fn 3.

10 Smith, Judicial Law Making in the Criminal Law, LQR 100 (1984), 46 (75), der deshalb für eine Kodifikation plädiert.

11 Vgl. Fourth Report on the Draft Code of Offences against the Peace and Security of Mankind, by Mr. Dodou Thiam, Special Rapporteur, Dok. A/CN.4/398 (11.3.1986), in: Yearbook of the International Law Commission (YbILC) 1986, Bd. II, Teil 1, 1988, S. 53, $70 \mathrm{ff}$. (para. 163); vgl. für die Diskussion innerhalb der ILC: YbILC 1986 Bd. I, S. 122, 140, 148, 150, 154, 156, 161, 164, 177; Bd. II 2, S. 50; 1988 II 2, S. 70

12 So die berühmte Formulierung im Bericht des UN-Generalsekretärs zur Errichtung des Jugoslawientribunals (Report of the Secretary General, UN-Doc. S/25704, para. 35).

13 S.W. v. UK, series A 335-b, Urteil v. 27.10.1995, para. 35; C.R. v. UK, series A 335-c, Urteil v. 27.10.1995, para. 33; Streletz, Kessler, Krenz v. Germany, Urteil v. 22.3.2001 (deutsch in NJ 2001, 261 = EuGRZ 2001, 210), para. 57; K.-H.W. v. Germany, ebd. 
crimen sine lege minus lex scripta, wobei freilich andere Autoren das »principle of legality « weniger systematisch definieren. ${ }^{15}$

Ohnehin wird der Nullum crimen Grundsatz im »common law « - hier verstanden als Gegenbegriff zum kontinentaleuropäischen »civil law« - weniger unter den lateinischen Begriff als vielmehr unter den schon genannten Begriff des »principle of legality « oder - noch weiter - der Rule of Law gebracht. In England hat er erst mit der Übernahme von Art. 7 EMRK durch den schon erwähnten Human Rights Act 1998 eine gesetzliche Grundlage erhalten, während etwa Art. 1 (9) cl. 3, (10) cl. 1 der USVerfassung die rückwirkende Schaffung von Straftatbeständen (sog. ex post facto legislation) explizit verbietet. ${ }^{16}$ Im englischen Recht kann es eine solche Regelung schon deshalb nicht geben, weil dort überhaupt keine geschriebene Verfassung existiert.

Ungeachtet dieser begrifflichen Fragen stellt sich das Sachproblem, inwieweit die überkommene Befugnis der Rechtsprechung, insbesondere des House of Lords, rechtssetzend - im Sinne des sog. Judicial Law Making - tätig zu werden, mit einem modernen, von Art. 7 EMRK gedeckten Verständnis des Nullum Crimen Grundsatzes vereinbar ist. Die Frage wurde im Jahre 1961 im Fall des Shaw ${ }^{17}$ relevant, der wegen der Veröffentlichung und Verbreitung eines sog. »Ladies Directory«, das Namen, Telefonnummern und Bilder von Damen zur Vermittlung sexueller Kontakte enthielt, u.a. wegen einer »conspiracy to corrupt public morals « angeklagt wurde. ${ }^{18}$ Der Streit entbrannte nun darum, ob ein solcher Straftatbestand überhaupt im Common Law existiere oder ob nicht vielmehr seine Anwendung eine rückwirkende Strafbegründung darstelle. Im Ergebnis haben die Lords die erstgenannte Ansicht vertreten, wobei in der Begründung deutlich wurde, dass sie durchaus an der traditionellen Rolle der Justiz als Sittenwächter (»Custos morum of the people«) festhalten wollten.

So hat Lord Viscount Simonds zwar bestritten, ein Vertreter des Judicial Law Making zu sein, zugleich aber den Gerichten eine »residual power to enforce the supreme and fundamental purpose of the law, to conserve ... the moral welfare of the state« zugeschrieben und sie in die Pflicht genommen »to guard it against attacks which maybe the more insidious because they are novel and unprepared for $\ll$. ${ }^{19}$ Damit hat er den Gerichten eine Auffang- bzw. Restzuständigkeit zur Vermeidung von Strafbarkeitslücken aus Gründen des überwiegenden Allgemeinwohls zugewiesen. So könne es etwa nicht hingenommen werden, dass mit der Legalisierung homosexueller Praktiken zwischen erwachsenen Männern auch die öffentliche Aufforderung zu solchen Praktiken straflos gestellt werde. In diesem Fall könne dem machtlosen Common

14 Ashworth, Law Quarterly Review (LQR) 107 (1991), 419 (442); ders., Principles, o. Fn. 5, S. 71.

15 Insoweit sei hier nur auf das grundlegende Werk von $R a z$ verwiesen, der die Rule of Law eher minimalistisch im Sinne eines Rechtssystems definiert hat, das von »rules which are fixed, knowable, and certain « beherrscht werde (The authority of law, 1997, S. 212 ff.).

16 Vgl. Boot, o. Fn. 1, S. 118 f.

17 Früher schon »Rex v. Manley«, 19331 K.B. 529 (1932), vgl. La Fave/Scott, o. Fn. 8, S. 65.

18 Shaw v. Director of Public Prosecutions (DPP), House of Lords, Urteil vom 4.5.1961, Appeal Cases (AC) 1962, 220.

19 Ebd., S. 267 f. 
Law nicht länger Referenz erwiesen werden; vielmehr hätten die Richter die Aufgabe, die vorhandenen Strafbarkeitslücken auszufüllen. ${ }^{20}$ Die anderen Richter haben sich diesem Votum - zum Teil unter Berufung auf anerkannte öffentliche Maßstäbe angeschlossen. ${ }^{21}$ Lediglich Lord Reid hat vor "public policy « Argumenten gewarnt und dafür plädiert, dem Parlament - als dem »proper place « - die Entscheidung solcher Wertungsfragen zu überlassen. ${ }^{22}$

Zehn Jahre später hatte das House of Lords in dem ähnlichen Fall Knuller darüber zu entscheiden, ob ein Magazin mit Anzeigen von Männern zur Vermittlung homosexueller Kontakte ebenfalls als »conspiracy to corrupt public morals« und als Verletzung der »public decency « anzusehen sei. ${ }^{23}$ Die Lords befanden sich nun in dem Dilemma, entweder die Bindungswirkung der Entscheidung in Shaw anzuerkennen und diese damit in der Sache nachträglich zu legitimieren, oder sie aufzuheben und sich damit erneut eine rechtssetzende Befugnis anzumaßen, anstatt mögliche Rechtsänderungen dem Parlament zu überlassen. Genau aus diesem Grund fühlte sich Lord Reid, der in Shaw die Mindermeinung vertreten hatte, an diese Entscheidung gebunden. ${ }^{24}$ Nur Lord Diplock kritisierte die Shaw-Entscheidung als eine $»$ judicial usurpation of ... an exclusively legislative power $\aleph^{25}$ und wollte sie als unzulässiges $\gg$ Judicial Law Making « aufheben. Dem ist die Mehrheit nicht gefolgt, hat aber immerhin im Wege eines obiter entschieden, dass die Shaw-Entscheidung nicht im Sinne einer gerichtlichen Auffangzuständigkeit zur Schaffung oder Erweiterung von Straftatbeständen interpretiert werden könne ${ }^{26}$ und ein solches »Judicial Law Making « heute auch nicht mehr zulässig sei. Zugleich wurde aber versucht, den Auslegungsspielraum der Gerichte - hinsichtlich der »unravished remnants« des common law - zu erweitern $^{27}$, indem der Bestimmtheitsgrundsatz relativiert wurde. ${ }^{28}$ Bestimmtheit im Sinne der genauen Voraussicht möglicher Konsequenzen menschlichen Handelns sei in keinem Rechtssystem möglich. Es gäbe kein Verfahren, mit dem mit absoluter Präzision vorausgesagt werden könne, wann eine Person strafrechtlich verantwortlich sei: »those who scate on thin ice can hardly expect to find a sign which will denote the precise spot where they may fall in. $\ll^{29}$ Diese von Lord Morris begründete Lehre des dünnen Eises hat Lord Simon of Glaisdale dann noch mit seiner Lehre vom risikoreichen Segeln am Wind ergänzt: »those who choose ... to sail as close as possible to the wind

20 Ebd., S. 268.

21 Vgl. ebd., 282 ff., 291 f., 292 f.

22 Ebd., 272 ff. (276).

23 Knuller v. DPP, Urteil House of Lords vom 14.06.1971, AC 1973, 435.

24 Ebd, S. 455 ff. (und deshalb nur der zweite Anklagepunkt hinsichtlich der Offence der public defency zurückzuweisen sei).

25 Ebd., 469 ff. (479). Zu diesem grds. Einwand auch Smith, o. Fn. 10, 67 ff.

26 Knuller, o. Fn. 23, 457 f., 464 f., 474, 490.

27 Vgl. ebd., $464 \mathrm{f}$.

28 I.E. ebenso Smith v. Donnelly, High Court of Justiciary (Schottland), Urteil v. 31.7.2001, 2001 S.L.T. 1007 u.a. bezugnehmend auf EGMR Steel v. UK, Urteil vom 23.09.1998, Reports 1998-VII

29 Knuller, o. Fn. 23, 463. Dazu auch Smith, o. Fn. 10, 71. 
inevitably run some risk $\ll^{30}$. Wer sich also in einer Grauzone zwischen Legalität und Illegalität bewege und sich damit dem Risiko einer Strafbarkeit aussetze, sei dafür selbst verantwortlich und könne sich später nicht darauf berufen, dass die einschlägigen Strafvorschriften den Bestimmtheitsgrundsatz verletzten. Dies erinnert an die versari-Haftung des alten kanonischen Kirchenrechts: versanti in re illicita imputantur omnia, quae sequuntur ex delicto. ${ }^{31}$ Im Übrigen gibt es angesichts der strukturell ähnlichen Relativierung des Bestimmtheitsgrundsatzes im deutschen Recht ${ }^{32}$ keinen Grund für kontinentaleuropäische Überheblichkeit gegenüber den common sense Argumenten der englischen Law Lords.

Wer freilich geglaubt hatte, mit Knuller habe das House of Lords seiner Judicial Law Making Befugnis endgültig abgeschworen, ${ }^{33}$ der sah sich spätestens im Jahre 1991 eines Besseren belehrt. War es bisher nur um die Schaffung neuer oder Erweiterung bestehender Tatbestände gegangen, so stellte sich nun die Frage, ob auch eine defence, also ein (materieller oder prozessualer) Straffreistellungsgrund, rückwirkend aufgehoben werden konnte. ${ }^{34}$ In Regina ${ }^{35}$ hatte ein Ehemann nach räumlicher Trennung von seiner Frau, aber noch vor Beginn des förmlichen Scheidungsverfahrens diese vergewaltigt. Die Lords hatten nun zu entscheiden, ob die - auf das Jahr 1736 zurückgehende ${ }^{36}$ - Common Law Regel, nach der eine Frau mit der Heirat unwiderruflich in den Geschlechtsverkehr mit dem Ehemann einwilligt, nach wie vor Gültigkeit habe. Bis zu Regina wurde in der Rechtsprechung die Auffassung vertreten, dass sich der Ehemann so lange auf eine »marital immunity « berufen könne, bis die Ehe geschieden oder eine entsprechende richterliche Verfügung erlassen worden sei. Dies ist nicht nur in der Literatur, sondern auch in einem working paper der Law Commission - mit dem bezeichneten Titel »rape within marriage « - heftig kritisiert und vorgeschlagen worden, diese Immunität abzuschaffen. ${ }^{37}$ Auch das House of Lords hat sich dieser Ansicht im Ergebnis mit dem Argument angeschlossen, dass die Ehe nach

30 Knuller, o. Fn. 23, 488.

31 Frei übersetzt: Wer eine unerlaubte Tat begeht, dem ist alles - auch Zufälliges - zuzurechnen, was aus dieser Tat folgt (vgl. Ambos, Präterintentionalität und Erfolgsqualifikation, GA 2002, 455 m.w.N.).

32 Vgl. etwa BVerfGE 14, 245 (251): »Die Gesetze würden sonst zu starr und kasuistisch und könnten der Vielgestaltigkeit des Lebens, dem Wandel der Verhältnisse oder der Besonderheit des Einzelfalls nicht mehr gerecht werden. Diese Gefahr läge nahe, wenn der Gesetzgeber stets jeden Tatbestand bis ins letzte ausführen müßte«. Vgl. jüngst auch Gusy, Verfassungsfragen des Strafprozessrechts, StV 2002, 153 (157), wonach das BVerfG Bestimmtheit immer mehr mit Bestimmbarkeit gleichsetze.

33 So wohl Smith, o. Fn. 10, 56: »judges will not now expressly claim a power to create new offences. « Vgl. aber die folgende Fn.

34 Diskutiert wurde bis dato allenfalls die rückwirkende Schaffung von defences, was ja an sich deshalb unproblematisch ist, weil sie zugunsten des Täters wirkt, vgl. Smith, o. Fn. 10, 63 ff.

$35 R$ v. R, Urteil v. 23.10.1991, AC 1992, 599.

36 Sir Matthew Hale CJ, History of the Pleas of the Crown: »but the husband can not be guilty of rape committed by himself upon his lawful wife, for by their matrimonial consent and contract the wife hath given up herself in this kind unto her husband, which she cannot retract.« (zitiert nach S.W. v. UK, o. Fn. 13, para. 10).

37 Vgl. Law Commission, Rape within marriage, working paper 116, para. 5.2. 
heutigem Verständnis einer Partnerschaft von Gleichberechtigten sei und sich das Common Law insoweit weiterentwickelt habe. ${ }^{38}$ Die entscheidende Frage sei deshalb, ob eine Einwilligung der Frau in den Geschlechtsverkehr zum Tatzeitpunkt tatsächlich noch bestanden habe; dies sei lediglich eine Beweisfrage. ${ }^{39}$

Ein weiteres Problem bestand aber darin, dass der Gesetzgeber des Jahres 1976 die Vergewaltigung als »unlawful sexual intercourse « definiert hatte, ${ }^{40}$ was von einigen dahingehend interpretiert wurde, dass erzwungener Geschlechtsverkehr nur außerhalb der Ehe als Vergewaltigung strafbar sei. Die Lords hielten den Begriff »unlawful« demgegenüber für überflüssig; die notwendige Beseitigung einer »common law fiction « könne er jedenfalls nicht verhindern. ${ }^{41}$ Diese ergebnis-orientierte Argumentation verträgt sich freilich kaum mit der Tatsache, dass der Gesetzgeber des Jahres $1994^{42}$ das Wort »unlawful« aus der Vergewaltigungsdefinition gestrichen hat, um damit endgültig klar zu machen, dass es nur auf die Einwilligung des Tatopfers ankomme. ${ }^{43}$

Im deutschen Recht existierte bekanntlich dasselbe Problem hinsichtlich der sexuellen Nötigung, war eine solche doch nach altem Recht (§ 178 StGB a.F.) - bis zur Änderung im Jahre $1997^{44}$ - nur bei »außerehelicher« Begehung strafbar, so dass eine Bestrafung innerehelicher Nötigungen gemäß 178 a.F. am Nullum crimen Grundsatz scheiterte.

Auch wenn man die englische »marital immunity « nicht als Tatbestandsausschluss bei ehelicher Vergewaltigung verstehen will, so läuft ihre rückwirkende Aufhebung doch auf die Strafbarstellung eines Verhaltens hinaus, das nach common law zum Zeitpunkt der Tatbegehung jedenfalls nicht bestraft werden konnte. Es wäre schon die Aufgabe des Gesetzgebers des Jahres 1976 - nicht erst 1994 - gewesen, durch Verzicht auf das Wort »unlawful« eindeutig klarzustellen, dass bei fehlender Einwilligung der Tatbestand der Vergewaltigung erfüllt ist. ${ }^{45}$ Auch ein subjektiviertes Verständnis des Nullum Crimen Grundsatzes im Sinne eines fair warning ${ }^{46}$ für den Bürger hilft hier kaum weiter, denn britische Ehemänner konnten sich bis zur Entscheidung in Regina immerhin auf eine mehr als 200-jährige Rechtssprechungspraxis, also auf gefestigtes Common Law, verlassen und hatten mangels parlamentarischer Initiativen auch keinen Anlass, von einer Rechtsänderung auszugehen. Ebenso klar ist freilich in der Logik des Common Law -, dass mit der Entscheidung in Regina eine spätere Berufung auf eine unwiderrufliche Einwilligung in den Geschlechtsverkehr durch Heirat ausgeschlossen war (und zwar noch vor der Gesetzesänderung im Jahre 1994).

$38 R v R$, o. Fn. 35, 616.

39 Ebd., $617 \mathrm{f}$.

$40 \gg .$. with a woman who at the time of the intercourse does not consent to it« (section 1 Abs. 1 Sexual Offences (Amendment) Act 1976).

$41 R v R$, o. Fn. 35, 621 ff. (623).

42 Criminal Justice and Public Order Act 1994.

43 Ebenso Giles, Judicial Law-Making in the Criminal Courts etc., CrimLR 1992, 407 (412).

44 6. Strafrechtsreformgesetz, BGBl. 1997 I, 164.

45 Für eine gesetzliche Lösung i.E. auch Giles, o. Fn. 43, 416 f.

46 Vgl. Ashworth, Principles, o. Fn. 5, S. 71, 74, 77; La Fave/Scott, o. Fn. 8, S. 71, 72. 
Unter Berufung auf Regina wurde deshalb auch zutreffend vom Court of Appeal in Elbekkay ${ }^{47}$ entschieden, dass eine erschlichene Einwilligung unwirksam sei, unabhängig davon, ob sich der Täter als Ehemann oder nur als Freund des Tatopfers ausgebe. Auch hier hatte das Gericht freilich wieder mit einer scheinbar entgegenstehenden statute rule zu kämpfen, hatte der Gesetzgeber des Jahres 1994 doch bei der Definition der Vergewaltigung nur den Fall geregelt, dass der Täter sich als Ehemann des Opfers ausgibt und den vorliegenden Fall, in dem das Opfer nur eine nichteheliche Beziehung hatte und sich der Täter als dessen nichtehelicher Partner ausgegeben hatte, ungeregelt gelassen. Der Court of Appeal fühlte sich - unter Berufung auf Regina - berufen, diese Lücke durch eine Gleichstellung zwischen Ehemann und nichtehelichem Partner zu schließen. Wie revolutionär diese Entscheidung war, zeigt sich etwa daran, dass der schottische High Court noch Mitte des $19 \mathrm{Jh}$. in Fraser ${ }^{48}$ auch die erschlichene Einwilligung durch den scheinbaren Ehemann als Strafausschlussgrund wertete.

Anders lag es im Fall des 12jährigen $C$., der eines versuchten Diebstahls angeklagt wurde, sich jedoch auf die sog. »presumption doli incapax«, also auf die Vermutung der Schuldunfähigkeit, berufen konnte. ${ }^{49}$ Der Court of Appeal hatte als Vorinstanz entschieden, dass diese Vermutung nicht länger Bestandteil des Common Law sei und deshalb C. verurteilt. Dem ist das House of Lords mit dem Argument entgegengetreten, dass ein Judicial Law Making grundsätzlich verboten sei und die rückwirkende Aufhebung einer solchen Schuldunfähigkeitsvermutung in die Zuständigkeit des Parlaments falle. Im Gegensatz zu Regina gehe es hier nicht um die Beseitigung einer »common law fiction $\ll .{ }^{50}$

Zusammenfassend lässt sich der dargestellten Rechtsprechung zwar eine deutliche Tendenz gegen die Schaffung neuer oder Erweiterung bestehender Straftatbestände entnehmen, doch scheint sich das Rückwirkungsverbot (noch) nicht als Bestandsschutz für im common law anerkannte defences durchgesetzt zu haben. Zudem ist schon auf Tatbestandsebene die Grenze zwischen unzulässiger richterlicher Rechtssetzung - Judicial Law Making - und zulässiger Auslegung bzw. Anwendung geltenden Rechts auf neue Sachverhalte - wie übrigens auch im Völkerstrafrecht im Hinblick auf Völkergewohnheitsrecht ${ }^{51}$ - nicht immer klar zu ziehen. ${ }^{52}$ Wenn, wie in C. v. DPP, Kriterien für das Judicial Law Making entwickelt werden, ${ }^{53}$ so bedeutet das nichts anderes als dass eine richterliche Rechtssetzungsbefugnis im Grundsatz immer noch anerkannt ist. ${ }^{54}$ Im schottischen Recht wird im Übrigen nach wie vor von einer »decla-

47 Criminal Law Review 1995, 163.

481847 Ark. 280; zit. nach Jones/Christie, Criminal Law, 2. Aufl. 1996, S. 24.

49 C (a minor) v. DPP, Urteil House of Lords 16.3.1995, AC 1996, 1.

50 Ebd., $37 \mathrm{f}$.

51 Vgl. schon Fn. 12 und dazugehöriger Text. Welche Normen in concreto völkergewohnheitsrechtlich anerkannt sind, ist freilich nicht immer einfach zu bestimmen, so dass die Grenzziehung zwischen der Schaffung von Strafnormen und der Anwendung bestehenden Völkergewohnheitsrechts schwierig ist.

52 Vgl. grdl. Smith, o. Fn. 10, 46; Ashworth, Interpreting criminal statutes etc. LQR 107 (1991), 419; auch La Fave/Scott, o. Fn. 8, S. 72; Jones/Christie, o. Fn. 48, S. 23 f. bezugnehmend auf die klassische Kritik von Lord Cockburn.

53 C. v. DPP, o. Fn. 49, S. 28 (Lord Lowry). 
ratory power« des High Court gesprochen und darunter nicht nur - wie der Begriff »declaratory« vermuten lässt - die Erklärung bzw. Auslegung bestehender Verbrechen, sondern auch die Schaffung neuer Tatbestände verstanden. ${ }^{55}$

Ist diese Praxis nun mit Art. 7 EMRK vereinbar?

\section{Art. 7 EMRK und common law}

Der EGMR hatte sich schon lange vor den Mauerschützenfällen mit Art. 7 EMRK zu befassen und zwar im Hinblick auf den Bestimmtheitsgrundsatz und das Rückwirkungsverbot. ${ }^{56}$ In Sunday Times $v . U K^{57}$ ging es um die Frage, ob das englische »contempt-Recht «, mit dem die Justiz vor Obstruktion und missbräuchlichen Angriffen (auch der Presse) geschützt werden soll ${ }^{58}$, eine zulässige Einschränkung der Meinungsfreiheit iSv. Art. 10 EMRK (»prescribed by law«) darstellt. Der Gerichtshof stellte zunächst klar, dass der Begriff »law « in der Konvention in allen Vorschriften gleich zu verstehen sei ${ }^{59}$ und auch common law einschließe, denn andernfalls wäre ein common law Staat vom Schutzbereich des Art. 10 EMRK ausgeschlossen und sein Rechtssystem selbst würde in Frage gestellt. ${ }^{60}$

Im Übrigen müsse das nationale Recht ausreichend zugänglich und so bestimmt sein, dass der Bürger - ggf. aufgrund entsprechenden Rechtsrats - in der Lage sei, es vorherzusehen und sein Verhalten danach auszurichten:

»... the law must be adequately accessible: the citizen must be able to have an indication that it is adequate in the circumstances of the legal rules applicable to a given case. ... a norm cannot be regarded as >law< unless it is formulated with a sufficient precision to enable the citizen to regulate his conduct: he must be able - if need be with appropriate advice - to foresee, to a degree that is reasonable in the circumstances, the consequences which a given action may entail. « ${ }^{61}$

54 Krit. auch Ashworth, Principles, o. Fn. 5, S 73.

55 Jones/Christie S. 18 ff.; auch Ashworth, Principles, o. Fn., S. 72.

56 Zusf. zur jüngeren Rspr. Cabral Barreto, La jurisprudence de la nouvelle Cour européenne des Droits de l'Homme sur l'article 7 de la Convention européenne de Droits de l'Homme, FS Trechsel, 2002, S. 3-16. Zum Verbot einer nachträglichen Strafverschärfung aufgrund Art. 7 EMRK (nulla poena sine lege) vgl. Ecer and Zeyrek v. Turkey, Urteil v. 27.2.2001, applicat. 29295/95 and 29363/95, para. $31 \mathrm{ff}$. (36 f.).

57 Sunday Times v. UK, Urteil v. 26.4.1979, Series A 30 (1979); dazu auch Smith, o. Fn. 10, 74.

58 Nach Sunday Times, o. Fn. 57, para. 18, sei das englische contempt Recht - unter Bezugnahme auf den Bericht des Committee on Contempt of Court ("Phillimore report") - »... "a means whereby the courts may act to prevent or punish conduct which tends to obstruct, prejudice or abuse the administration of justice either in relation to a particular case or generally' and as existing to protect not the dignity of the judges but 'the administration of justice' and 'the fundamental supremacy of the law' «.

59 Ebd., para. 40; ebenso S.W. v. UK, o. Fn. 13, para. 35; C.R. v. UK, o. Fn. 13, para. 33; Streletz et. al. v. Germany, o. Fn. 13, para. 57; K.-H.W. v. Germany, o. Fn. 13, para. 62.

60 Sunday Times v. UK, o. Fn. 57, para. 40: »would deprive a common-law-state which is party to the Convention of the protection of Art. 10 and strike at the very roots of that State's legal system «

61 Ebd., para. 49. 
Kann diese Ansicht als st. Rspr. bezeichnet werden, ${ }^{62}$ so redet der Gerichtshof andererseits einer Relativierung des Bestimmtheitsgrundsatzes und des Analogieverbots das Wort, wenn er - schon in Sunday Times - feststellt, dass »absolute Bestimmtheit « unerreichbar sei und das Recht mit den wechselnden Umständen Schritt halten müsse. So sei es unvermeidbar, dass zahlreiche Gesetze mehr oder weniger vage gefasst seien und ihre Auslegung eine Frage der Praxis sei. ${ }^{63}$ In ähnlicher Weise hat der Gerichtshof in Kokkinakis v. Greece ${ }^{64}-$ hier ging es wieder um den Begriff »law«, diesmal in Art. 9 EMRK - und in Baskaya and Okcuoglu v. Turkey $^{65}$ (Urteil v. 8.7.1999) - Verurteilung der Beschwerdeführer aufgrund des türkischen Antiterrorismusgesetzes wegen »dissemination of propaganda against the indivisibility of the State « - die Bestimmtheitsanforderungen relativiert.

In unserem Zusammenhang besonders relevant ist dabei, dass der Gerichtshof auch die ständige Rechtsprechung zur Feststellung ausreichender Bestimmtheit und Vorhersehbarkeit i.S.v. Art. 7 EMRK heranzieht. ${ }^{66}$ So hat er unter Bezugnahme auf die st. Rspr. des französischen Kassationsgerichtshofs, die veröffentlicht und damit zugänglich sei, die Verurteilung eines Fahrlehrers wegen sexueller Nötigung einer Fahrschülerin aufrechterhalten, obwohl die Tat erst durch eine spätere Gesetzesänderung als »contrainte « strafbar gestellt wurde. ${ }^{67}$ In Steel v. UK hat der Gerichtshof den engli-

62 Vgl. Kokkinakis v. Greece, Urteil v. 19.04.1993, Series A 260 a, para. 52; S.W. v. UK, o. Fn. 13, para. 35; C.R. v. UK, o. Fn. 13, para. 33; Steel v. UK, o. Fn. 28, para. 54. Bei der Verbreitung von Texten hänge die Vorhersehbarkeit der Bestrafung von dem in Rede stehenden Text, seinem Verbreitungsgrad und Zahl und Status der Adressaten ab (Groppera Radio AG and Others v. Switzerland, Urteil v. 28.3.1990, Ser. A 173, para. 68; auch Cantoni v. France, Urteil v. 15.11.1996, Reports 1996-V, para. 35). Vorhersehbarkeit sei auch bei der Notwendigkeit der Einholung von Rechtsrat gegeben (ebd., para. 35; Miloslavsky v. U.K., Urteil v. 13.7.1995, Ser. A 316-B, para 37), insbes. bei Berufsgruppen, die hohe Sorgfalt walten lassen müssen (Cantoni v. France, para. 35). - Vgl. auch Kommission in G. v. Germany, Appl. No. 13079/87, 6.3.1989, 60 DR 256 (261 f.).

63 Sunday Times, o. Fn. 57, para. 49: »Those consequences need not be foreseeable with absolute certainty: experience shows this to be unattainable. Again, whilst certainty is highly desirable, it may bring in its train excessive rigidity and the law must be able to keep pace with changing circumstances. Accordingly, many laws are inevitably couched in terms which, to a greater or lesser extent, are vague and whose interpretation and application are questions of practice."

64 Kokkinakis v. Greece, o. Fn. 62, para. 40: »the wording of many statutes is not absolutely precise. The need to avoid excessive rigidity and to keep pace with changing circumstances means that many laws are inevitably couched in terms which, to greater or lesser extent, are vague ...«.

65 Baskaya and Okcuoglu v. Turkey, Urteil v. 8.7.1999, Reports 1999-IV, insbes. para. 39. Der Gerichtshof hielt diese Verurteilung für vorhersehbar, weil im Abs. 2 der entspr. Vorschrift die strafbaren Veröffentlichungen aufgeführt wurden, bejahte jedoch eine Verletzung von Art. 10 EMRK. Im Übrigen sei absolute Bestimmtheit schwierig und ein bestimmter Grad von Flexibilität zulässig; dazu auch Boot, o. Fn. 1, $150 \mathrm{f}$.

66 Vgl. neben den folgenden Urteilen auch Baskaya and Okcuoglu v. Turkey, para. 39. Vgl. insoweit auch das Urteil des BVerfG zur Vermögensstrafe: Urteil vom 20.03.2002, Az. 2 BvR 794/95.

67 G. v. France, Urteil v. 27.9.1995, Ser. A 325-B, para. 25: »... the offences ... fell within the scope of the former Art. 332 and 333 of the Criminal Code, which satisfied the requirements of foreseeability and accessibility ... there was consistent case law from the Court of 
schen Tatbestand des »Friedensbruchs « (breach of the peace) für ausreichend bestimmt gehalten, weil die englische Rspr. der letzten zwei Jahrzehnte ihn klar definiert habe; er liege nämlich bei der (wahrscheinlichen) Verletzung von Rechtsgütern von Personen und Eigentum, die Dritte zu Gewaltakten verleiten, vor. ${ }^{68}$

Es trägt freilich nicht zur Bestimmtheit und Vorhersehbarkeit dieses Tatbestandes bei, dass er in Schottland weiter, nämlich ohne die Voraussetzung einer Rechtsgutsverletzung (»harm principle«), verstanden wird. ${ }^{69}$

Mit ähnlicher Begründung hat der Gerichtshof die Aufhebung der ehelichen Immunität in Regina und anderen unterinstanzlichen Entscheidungen mit Art. 7 EMRK für vereinbar gehalten. ${ }^{70}$ In jedem Strafrechtssystem existiere ein »inevitable element of judicial interpretation «. In England sei die progressive Entwicklung des Strafrechts durch judicial lawmaking notwendiger Teil der Rechtstradition und solch eine »judicial interpretation « (!) mit Art. 7 EMRK vereinbar, wenn das Auslegungsergebnis dem Wesen der Straftat entspreche und vernünftigerweise vorhergesehen werden konnte. ${ }^{71}$ In concreto sei die Aufhebung der immunity-Regel nicht mehr als die Folge einer "perceptible line of case law development « und ein »reasonably foreseeable development « gewesen, das dem Wesen des Straftatbestands der Vergewaltigung entspreche. ${ }^{72}$ Die Strafbarstellung der Vergewaltigung sei im Übrigen auch mit Gegenstand und Zweck von Art. 7 EMRK - der Verhinderung willkürlicher Strafverfolgung - vereinbar. Die Aufgabe der Immunität sei durch ein »zivilisiertes« Verständnis der Ehe und die grundlegenden Ziele der Menschenrechtskonvention - Schutz menschlicher Würde und Freiheit - sogar gefordert. ${ }^{73}$

Der Gerichtshof geht damit zwar über ein bloß subjektives, schuldstrafrechtliches Verständnis des Nullum crimen Grundsatzes hinaus, indem er Vorhersehbarkeit objektiviert und auf eine schutzzweckorientierte, teleologische Auslegung von Art. 7 EMRK und der Menschenrechtskonvention als Ganzes rekurriert. An die Stelle der Einzelfallprüfung treten jedoch allgemeine policy-Erwägungen und kriminalpolitische Wertungen, wie sie im englischen Recht als »social defence-Argument« oder »purposive approach« bekannt sind. ${ }^{74}$ Sie machen die Entscheidung scheinbar gegen Kritik

Cassation, which was published and therefore accessible .... As regards the notion of violence, the new provisions in the new art. 332 and $333 \ldots$ merely confirm this case law." (Herv. d. Verf.); dazu auch Boot, o. Fn. 1, 149 f.

68 Steel v. UK, o. Fn. 28, para. 55: »... breach of the peace is committed only when an individual causes harm, or appears likely to cause harm, to persons or property or acts in a manner the natural consequences of which would be to provoke others to violence. .... Accordingly, the Court considers that the relevant legal rules provided sufficient guidance and were formulated with the degree of precision required by the Convention «. Krit. dazu Ashworth, Principles, o. Fn. 5, S. 77.

69 Vgl. Smith v. Donnelly, o. Fn. 28, para. 10 ff. (16).

70 S.W. v. UK, o. Fn. 13, para. 34 ff.; C.R. v. UK, o. Fn. 13, para. $32 \mathrm{ff}$.

71 Ebd., para. 36 und 34: »consistent with the essence of the offence and could reasonable be foreseen $\ll$.

72 Ebd., para. 43 und 41.

73 Ebd., para. 44 und 42.

74 Vgl. Smith, o. Fn. 10, 52; Ashworth, Principles, o. Fn. 5, S. 78 ff., 82 ff. 
immun, müssen aber doch dann versagen, wenn ein Urteil zwischen richtig und falsch nicht so eindeutig wie in Regina getroffen werden kann.

\section{Art. 7 EMRK, Art. 103 II GG und die Mauerschützen}

Im Verhältnis zu Art. 103 II GG wird Art. 7 EMRK einerseits weniger strikt verstanden, nämlich im Hinblick auf das Analogieverbot (lex stricta) ${ }^{75}$ und vor allem die Zulässigkeit völkergewohnheitsrechtlicher Bestrafung (lex scripta), ${ }^{76}$ wobei diese schon auf Art. 7 Abs. 1 - Strafbarkeit nach »internationalem Recht« -, nicht erst auf die sog. Nürnberg-Klausel des Abs. 2 gestützt werden kann; die Nürnberg-Klausel stellt vielmehr eine echte Ausnahme vom Rückwirkungsverbot dar, weil sie eine Rückwirkung bei internationalen Verbrechen erlaubt. ${ }^{77}$ Andererseits geht Art. 7 EMRK aber über Art. 103 II GG hinaus, weil der EGMR den Schuldgrundsatz in Art. 7 EMRK hineinliest, der ja bekanntlich in Deutschland aus Art. 1 I, 2 I, 20 III GG abgeleitet wird. ${ }^{78}$ Freilich hätte das weitere Verständnis von Art. 7 EMRK nur dann unmittelbare Auswirkungen auf Art. 103 II GG, wenn Art. 7 EMRK nicht nur Gesetzesrang (Art. $59 \mathrm{GG}$ ) hätte, ${ }^{79}$ sondern gegenüber Art. 103 II GG vorrangig wäre. So bleibt es der Rspr. überlassen, ob und inwieweit Art. 7 EMRK die Auslegung von Art. 103 II GG beeinflusst. Der Gerichtshof jedenfalls billigt das common law Verständnis des Gesetzlichkeitsprinzips, er macht damit den kleinsten gemeinsamen Nenner zum europaweit gültigen Standard.

Wenn man die dargestellte Rechtsprechung des EGMR noch einmal Revue passieren lässt, vor allem auch im Hinblick auf die Achtung nationaler kriminalpolitischer Wertentscheidungen, sei es der Legislative oder Judikative, ${ }^{80}$ so überrascht es nicht, dass der Gerichtshof in den Mauerschützenverfahren einen Verstoß gegen Art. 7 EMRK verneint hat. Man mag dies im Ergebnis begrüßen, doch sieht sich die Urteilsbegründung erheblichen Einwänden ausgesetzt: ${ }^{81}$

75 Vgl. BVerfGE 71, 108 (115): ausgeschlossen ist »jede Rechts-'Anwendung', die über den Inhalt einer gesetzlichen Sanktionsnorm hinausgeht ... Der mögliche Wortsinn ... markiert die äußerste Grenze zulässiger richterlicher Interpretation ...«

76 Vgl. auch Arnold/Karsten/Kreicker, Menschenrechtsschutz durch Art. 7 Abs. 1 EMRK, NJ 2001, 561 (562); Ebert, Völkerstrafrecht und Gesetzlichkeitsprinzip, FS Müller-Dietz, 2001, S. 171-185, 181 ff. (182); Kreicker, Art. 7 EMRK und die Gewalttaten an der deutsch-deutschen Grenze, 2002, S. 9 ff.

77 Vgl. Kreicker, o. Fn. 76, S. 96 ff.

78 Vgl. Arnold/Karsten/Kreicker, o. Fn. 76, 567; Kreicker, o. Fn. 76, S. 49 ff. (51).

79 Der innerstaatliche Rang eines völkerrechtlichen Vertrags richtet sich nach dem Rang des ZustimmungsG (Schweitzer, Staatsrecht III, 7. Aufl. 2000, Rn. 447, 709). BGBl 1952 II, 686 spricht insoweit von »Gesetzeskraft«. Vgl. auch BVerwG DÖV 1998, 924, 925: »Der europäischen Menschenrechtskonvention kommt nur der Rang eines einfachen Bundesgesetzes zu. ... Eine konventionswidrige Auslegung stellt sich demnach als eine unrichtige Anwendung einfachen Bundesrechts dar.«.

80 Ebd., S.W. v. UK, o. Fn. 13, para. 42; C.R. v. UK, , o. Fn. 13, para 40.

81 Krit. auch Arnold/Karsten/Kreicker, o. Fn. 76, 561; Rau, Deutsche Vergangenheitsbewältigung vor dem EGMR etc., NJW 2001, 3008; Kreicker, o. Fn. , S. 5 m.w.N. in Fn. 21 und passim; Lensing/Mertens, Der EGMR und die »Mauerschützen«, in: Institut für Juristi- 
- Der Gerichtshof weicht dem schwierigen Problem der rückwirkenden Außerkraftsetzung von $\S 27$ DDR Grenzgesetz dadurch aus, dass er die vorliegenden Fälle nicht als Verbrechen gemäß $§ 213$ Abs. 3 DDR-StGB einstuft und deshalb § 27 GrenzG schon gar nicht für anwendbar hält. ${ }^{82}$

- Der Gerichtshof billigt es einem Rechtsstaat, der an die Stelle eines früheren Unrechtsstaats getreten ist, ohne weiteres zu, dass Recht des untergegangenen Staates nach seinen Grundsätzen auszulegen. ${ }^{83}$ Dabei ist unklar, von welchem Nullum crimen Verständnis der Gerichtshof ausgeht. Wenn man das zur Tatzeit geltende Recht mit Hilfe späterer, zudem in einem anderen - nicht rechtsstaatlichen! - System geltender Rechtsprinzipien auslegen will, so fragt es sich, was dann noch vom Tatzeitrecht, also dem Recht des Tatorts im gegebenen Systemzusammenhang, übrig bleibt. Tatsächlich geht der Gerichtshof von einem westlich vorgeprägten Rechtsstaatsverständnis aus und betrachtet sozialistisches Recht schon gar nicht als Recht im Sinne von Art. 7 der EMRK. Das wird besonders deutlich, wenn er an anderer Stelle davon spricht, dass die DDR Staatspraxis nicht als Recht in diesem Sinne angesehen werden kann. ${ }^{84}$ Man mag dem im Ergebnis zustimmen. Eine solche »westliche« Auslegung von Tatortnormen dürfte aber kaum mit einem Nullum crimen Grundsatz vereinbar sein, der - wertneutral - universelle Gültigkeit beansprucht und deshalb nach systemimmanenter Fremdrechtsanwendung verlangt; ${ }^{85}$ andernfalls wird er durch eine verdeckte Rückwirkung verletzt. ${ }^{86}$

- Wenn man das Kriterium der Vorhersehbarkeit oder des fair warning, das der Gerichtshof ja schon in der früheren Rechtsprechung entwickelt hat, ${ }^{87}$ ernst nimmt, so kann es nicht darum gehen, ob die Angeklagten die Menschenrechtswidrigkeit/Strafbarkeit ihrer Handlungen vorhersehen konnten, sondern vielmehr nur darum, ob sie $e x$ ante damit rechnen konnten, dass diese Handlungen ex post als strafbar bewertet würden. ${ }^{88}$ Wenn man die Verneinung dieser Frage für unerträglich hält, so sollte man ehrlicherweise den Nullum crimen Grundsatz a limine beschränken und ihn - als bloßes

sche Zeitgeschichte (Hrsg.), Jahrbuch für Juristische Zeitgeschichte, Band 3, Baden-Baden 2002, S. 352 (364 ff.); zust. etwa Starck, JZ 2002, 1102; Hassemer, Naturrecht im Verfassungsrecht, FS Trechsel, 2002, S. 135-150 (149); Naucke, Bürgerliche Kriminalität, Staatskriminalität und Rückwirkungsverbot, FS Trechsel, 2002, S. 505-516 (509, 511); i. E. zust. Werle, Rückwirkungsverbot und Staatskriminalität, NJW 2001, 3001 (3003ff.).

82 Streletz et al. v. Germany, o. Fn. 13, para. 56 ff. (63); krit. hierzu Arnold/Karsten/Kreicker, o. Fn. 76, 563; explizit zust. aber Naucke, o. Fn. 81, S. 509.

83 Streletz et al. v. Germany, o. Fn. 13, para 81. Ebenso Glässner v. Germany, Beschwerde 46362/99, Entscheidung v. 28.06.2001, merits.

84 Streletz et al. v. Germany, o. Fn. 13, para 87.

85 Krit. auch Arnold/Karsten/Kreicker, o. Fn. 76, 563, 565; Rau, o. Fn. 81, 3010; Kreicker, o. Fn. 76, S. 23.

86 Vgl. schon Alexy, Der Beschluss des BVerfG zu den Tötungen an der innerdeutschen Grenze, 1997, S. 8; ders, Mauerschützen. Zum Verhältnis von Recht, Moral und Strafbarkeit, 1993, S. 30; zust. bzgl. EGMR Rau, o. Fn. 81, 3010.

87 Und dem auch das $B$ VerfG - unter Berücksichtigung der st. Rspr. als Rechtsquelle (dazu schon o. Fn. 66 und dazugehöriger Text) - gefolgt: BVerfGE 26, 42; 47, 109 (120); 71, 108 (115); vgl. auch BGHSt 28, 312 (313).

88 Arnold/Karsten/Kreicker, o. Fn. 76, 566. 
Prinzip statt als Regel ${ }^{89}$ - einer Interessenabwägung - Rechtssicherheit v. Gerechtigkeit - unterwerfen. Das ist der überzeugendere, weil ehrlichere, völkerstrafrechtlichnaturrechtliche Ansatz, der auf ein nullum crimen sine iure hinausläuft. ${ }^{90}$ Will man nicht ohnehin so weit gehen wie das amerikanische Militärtribunal im Juristenurteil und das Rückwirkungsverbot nicht auf Völkergewohnheitsrecht oder common law anwenden, ${ }^{91}$ so kommt es letztlich darauf an, ob eine Bestrafung aus Gerechtigkeitsgründen unabdingbar erscheint. Im innerstaatlichen Recht würde dieser Ansatz freilich eine - schon früher geforderte ${ }^{92}$ - Änderung von Art. 103 II GG voraussetzen, ${ }^{93}$ die sich beim gegenwärtigen Stand des Völkerstrafrechts auf die im Rom-Statut ${ }^{94}$ anerkannten und durch das deutsche Völkerstrafgesetzbuch ${ }^{95}$ innerstaatlich umgesetzen völkerrechtlichen Kernverbrechen (Genozid, Verbrechen gegen die Menschlichkeit, Kriegsverbrechen) - als »minimum standard« - beschränken kann und damit die rechtsstaatlich problematische Unbestimmtheit der »Nürnberg-Klausel« (Art. 7 II EMRK, Art. 15 II IPbpR) überwinden würde.

- Der Gerichtshof verfasst zwar zwei Urteile zu den Funktionsträgern einerseits und zu dem Grenzsoldaten K.-H.W. andererseits, zieht daraus aber nicht die vom Schuldgrundsatz gebotene Konsequenz und verneint die Vorhersehbarkeit der Strafbarkeit im Fall des Grenzsoldaten auf der Grundlage eines unvermeidbaren Verbotsirrtums. ${ }^{96}$

89 Alexy, o. Fn. 86 (Beschluss), S. 23 f. Aus angloamerikanischer Sicht grdl. Dworkin, The model of rules, U. Chicago Law Review 35 (1967), 22-29.

90 Grdl. Kelsen, ICLQ 1 (1947), 153, 165; ebenso Boot, o. Fn. 1, S. 218. Im völkerstrafrechtlichen Sinne auch Arnold/Karsten/Kreicker, o. Fn. 76, 565 f., wenn sie eine (naturrechtliche) Menschen- und Völkerrechtswidrigkeit der Todesschüsse annehmen wollen. Für eine explizit naturrechtliche Begründung Naucke, o. Fn. 81, S. 513 ff. Werle, o. Fn. 81, 3003 ff., qualifiziert die Tötungen an der Grenze sogar als Verbrechen gegen die Menschlichkeit (3005 f.); ebenso das Sondervotum von Richter Loucaides (Zypern), EuGRZ 2001, 219 (221 f.) = NJ 2001, 270 f.; tendenziell auch Rau, o. Fn. 81, 3013; Lensing/Mertens, o. Fn. 81, S. 367; krit. zuletzt Ebert, o. Fn. 76, S. 178 m.w.N.

91 U.S. v. Altstoetter et al., Trial of War Criminals III, 975: »... sheer absurdity to suggest that the ex post facto rule ... could be applied to a treaty, a custom, or a common law decision of an international tribunal ... to apply the ... principle to judicial decisions of common international law would ... strangle that law at birth«. Vgl. auch Kelsen, ICLQ 1 (1947), 153, 165: Rückwirkungsverbot »does not apply to customary law and to law created by precedent, for such law is necessarily retroactive in respect to the first case to which it is applied."

92 Vgl. dafür schon Ambos, Nuremberg revisited, Strafverteidiger 1997, 39 (42).

93 Dafür jüngst auch Ebert, o. Fn. 76, S. $184 \mathrm{ff}$.

94 O. Fn. 3, Art. 5-8.

95 BGB1. 2002 I, 2254. Zum Entwurf der Expertenarbeitsgruppe vgl. BMJ (Hrsg.), Arbeitsentwurf eines Gesetzes zur Einführung des Völkerstrafgesetzbuchs - mit Begründung, 2001. Vgl. auch Kreß, Vom Nutzen eines deutschen Völkerstrafgesetzbuchs, 2000; Werle, Konturen eines deutschen Völkerstrafrechts, JZ 2001, 885; Zimmermann, Auf dem Weg zu einem deutschen VStGB, ZRP 2002, 97; Satzger, Das neue VStGB, NStZ 2002, 125; Werle/Geßßberger, Das Völkerstrafgesetzbuch, JZ 2002, 725.

96 So zutreffend die Sondervoten der Richter Cabral Barreto (Portugal), NJ 2001, $272=$ EuGRZ 2001, 219 (222 f.) und Pellonpää/Zupancic (Finnland/Slowenien), NJ 2001, 272 f., 273 f. = EuGRZ 2001, 219 (223 f.). Vgl. auch Arnold/Karsten/Kreicker, o. Fn. 76, 68 f.; Kreicker, o. Fn. 76, S. 56 ff.; Lensing/Mertens, o. Fn. 81, S. 368 f. 